\title{
O acervo Helena Antipoff como laboratório de pesquisa sobre a história das Ciências da Educação
}

\section{The Helena Antipoff archives as a laboratory of research on the history of the Sciences of Education}

\author{
Regina Helena de Freitas Campos*
}

\begin{abstract}
RESUMO
$\mathrm{O}$ artigo relata atividades de pesquisa desenvolvidas a partir do acervo documental do Centro de Documentação e Pesquisa Helena Antipoff, instituição criada em 1980 com a finalidade de preservar a memória e de divulgar a obra da psicóloga e educadora russo-brasileira Helena Antipoff (1892-1974). A riqueza e a diversidade do acervo, que documenta capítulos importantes da construção e circulação das ciências da educação no século 20, no âmbito das relações entre a Europa e o Brasil, favorecem a realização de pesquisas sobre o desenvolvimento teórico-prático da psicologia e das ciências da educação, assim como sobre os trabalhos realizados nas inúmeras instituições criadas e orientadas por Antipoff no Brasil. São referenciados trabalhos de pesquisa conceitual, sobre a história de modelos de trabalho em psicologia desenvolvidos em instituições educacionais criadas sob a liderança de Antipoff e trabalhos sobre formação de educadores na tradição antipoffiana. Conclui-se que a realização desses estudos contribui para o melhor conhecimento da história da psicologia e das ciências da educação e de sua circulação internacional, fornecendo modelos relevantes para se pensar o desenvolvimento contemporâneo dessas áreas científicas.
\end{abstract}

Palavras-chave: Helena Antipoff. Ciências da Educação. Circulação de ideias. Escola Nova. Pesquisa em ciências da educação.

* Universidade do Estado de Minas Gerais. Programa de Pós-Graduação em Educação. Belo Horizonte, Minas Gerais, Brasil. E-mail: regihfc@terra.com.br. http://orcid.org/0000-00016228-7076. 


\begin{abstract}
The article reports on research activities developed from the documentary archive of the Helena Antipoff Documentation and Research Center, an institution created in 1980 with the purpose of preserving memory and disseminating the work of the Russian-Brazilian psychologist and educator Helena Antipoff (1892-1974). The richness and diversity of the collection, which documents important chapters on the construction and circulation of education sciences in the 20th century within the framework of the relations between Europe and Brazil, favor research on the theoretical and practical development of psychology and education, and on the work done in the numerous institutions created and directed by Antipoff in Brazil. Research on the history of concepts and work models in psychology developed in educational institutions created under the leadership of Antipoff and work on the training of educators in the antipoffian tradition are reported. It is concluded that the accomplishment of these studies contributes to a better understanding of the history of psychology and education sciences and their international circulation, providing relevant models for thinking about the contemporary development of these scientific areas.
\end{abstract}

Keywords: Helena Antipoff. Sciences of education. Circulation of ideas. New Education. Research on the sciences of education.

O Centro de Documentação e Pesquisa Helena Antipoff (CDPHA) foi fundado em 25 de março de 1980 por um grupo de familiares, amigos e colaboradores de Helena Antipoff (1892-1974), psicóloga e educadora russo-brasileira que participou do movimento da Escola Nova e da construção das modernas ciências da educação na Europa. Ela é reconhecida nacional e internacionalmente por seu trabalho pioneiro na construção de perspectivas inovadoras na educação brasileira, em especial nas áreas da psicologia da educação, da pedagogia experimental, da educação especial e da educação rural.

Os objetivos do CDPHA, definidos em Estatuto, incluem a preservação da memória e do acervo da educadora, bem como a divulgação de sua obra. Entre as atividades do CDPHA, desde sua fundação, destacam-se a realização dos Encontros Anuais Helena Antipoff (cuja trigésima sexta edição foi realizada em abril de 2018), a publicação do Boletim do CDPHA, com informações sobre os encontros anuais (que focalizam temáticas relevantes na obra de Antipoff), e a edição da Coleção Encontros Anuais Helena Antipoff, em que são divulgados trabalhos selecionados apresentados nos eventos (CAMPOS; VIEIRA, 2007; NASSIF; NUNES, 2008; LOURENÇO; GUEDES; CAMPOS, 2009; SANTIAGO; CAMPOS, 2011; LOURENÇO, ASSIS; CAMPOS, 2012; ASSIS; LOURENÇO; BORGES, 2015; CAMPOS; DUARTE; CASSEMIRO, 2017). 
Uma das principais atividades atuais do CDPHA é a preservação e a disponibilização para a pesquisa do acervo documental que pertenceu à educadora, atualmente guardado em duas seções: na Sala Helena Antipoff, na Biblioteca Central da UFMG - Universidade Federal de Minas Gerais, e na Fundação Estadual Helena Antipoff, localizada no município de Ibirité, Minas Gerais, onde atualmente está sediado o Campus Ibirité da Universidade do Estado de Minas Gerais. $\mathrm{O}$ acervo inclui documentos relacionados à trajetória de Helena Antipoff: os estudos em centros importantes na construção das modernas ciências da educação em Paris (Laboratório Binet-Simon) e Genebra (Instituto Jean Jacques Rousseau de Ciências da Educação), o trabalho como psicóloga e educadora na Rússia, na Suíça e no Brasil, assim como se refere à organização e ao funcionamento das Sociedades Pestalozzi e do Complexo Educacional da Fazenda do Rosário, na cidade de Ibirité, Minas Gerais. Vale mencionar que esses documentos foram elaborados e acumulados no período de 1912 até a atualidade, incluindo também os que foram elaborados pelo próprio CDPHA.

Por sua riqueza e diversidade, e por seu valor na preservação da memória sobre aspectos relevantes da elaboração e circulação do movimento da Escola Nova e de construção das modernas ciências da educação, da pedagogia experimental e de modelos de educação especial em diferentes contextos, que fazem parte da própria história cultural e social do século 20, tanto em termos nacionais quanto internacionais, o acervo Antipoff tem despertado a curiosidade e inspirado trabalhos de investigação de pesquisadores brasileiros e estrangeiros.

Sabemos que, nos últimos anos, a pesquisa sobre o movimento da Escola Nova e seus desdobramentos na construção das ciências da educação têm chamado a atenção de historiadores e estudiosos por sua relação com as problemáticas da educação contemporânea. Entre essas problemáticas, destacam-se as tensões entre as exigências escolares e os ritmos de desenvolvimento da criança, a adequação de métodos pedagógicos às expectativas dos alunos e das famílias, polêmicas nos processos de avaliação escolar, as questões relacionadas à ética, à violência, aos processos de inclusão e à aceitação das diferenças no ambiente escolar, etc. Algumas propostas do movimento escolanovista ressurgem nas discussões atuais para a formação escolar de crianças e jovens, e a pesquisa sobre suas origens evoca interesse renovado (GUTIERREZ, 2011). Por esses motivos, o acervo do CDPHA tem alimentado a pesquisa sobre a história da psicologia e das ciências da educação e o intercâmbio acadêmico e científico com centros de pesquisa similares no Brasil e no exterior ${ }^{1}$.

1 O CDPHA tem mantido relações de cooperação acadêmica e científica no exterior com os Archives de l'Institut Jean-Jacques Rousseau e os Archives Piaget, na Faculdade de Psicologia e Ciências da Educação da Universidade de Genebra; Center for the History of Psychology, Akron 
Este artigo tem por objetivo apresentar como tem se desenvolvido a pesquisa, com base no acervo do CDPHA, e as perspectivas de intercâmbio e de cooperação que têm sido abertas, visando ao avanço do conhecimento sobre a história das ciências da educação e seus desdobramentos contemporâneos.

\section{Helena Antipoff, a pesquisa educacional e a circulação das ciências da educação no século 20}

A psicóloga e educadora Helena Antipoff foi uma personagem ímpar na história da educação brasileira. Psicóloga, pesquisadora, professora, ela fundou diversas instituições; publicou artigos, militou em prol dos direitos da criança e ajudou a escrever a história da educação e das ciências da educação tanto no Brasil quanto em termos internacionais. Nascida em Grodno, na Rússia czarista, viveu com sua família em São Petersburgo até 1909, ano em que se transferiu para Paris, onde iniciou estudos de nível superior. Entre 1911 e 1912, estagiou no laboratório de psicologia pedagógica instalado na Sorbonne pelo psicólogo Alfred Binet (1857-1911), participando da investigação sobre níveis de desenvolvimento mental de escolares parisienses sob a orientação do médico psiquiatra Théodore Simon (1873-1961), companheiro de Binet no desenvolvimento das primeiras escalas de medida objetiva da inteligência então inventadas. Entre 1912 e 1914, a convite do médico e psicólogo suíço Édouard Claparède (1873-1940), transferiu-se para Genebra, onde se diplomou como educadora no Instituto Jean-Jacques Rousseau de Ciências da Educação. Voltou à Rússia em 1916, tendo então trabalhado como psicóloga e educadora em instituições de acolhimento de crianças e adolescentes abandonados e em situação de risco social, nas cidades de São Petersburgo² e Viatka ${ }^{3}$. Em 1924, deixou a Rússia acompanhando o marido, então exilado em Berlim, e, em 1926,

University, EUA; Centre Alexandre Koyré d'Histoire des Sciences et des Techniques, École des Hautes Études em Sciences Sociales, Paris, França; Centro Alexandre Solzenitcyn de Estudos da Diáspora Russa, Moscou, Rússia. No Brasil, mantém relações de cooperação com grupos de estudo da história da psicologia em diversas universidades (USP, UFRJ, UERJ, PUC-SP, UNB, UFRGS, entre outras), reunidos na Sociedade Brasileira de História da Psicologia.

2 A cidade de São Petersburgo, localizada na parte mais ocidental da Rússia, à beira do Mar Báltico, foi sede do império czarista até 1917, ano da Revolução Comunista. Em 1914, passou a ser denominada Petrogrado. Em 1924, o nome foi outra vez mudado para Leningrado. Após a queda do regime comunista voltou ao nome original. É conhecida até a atualidade como a capital cultural do país.

3 Cidade localizada na região central da Rússia Europeia, atualmente denominada Kirov. 
voltou a Genebra, onde se tornou assistente de Claparède no Instituto Rousseau e no Laboratório de Psicologia da Universidade de Genebra.

\section{FIGURA 1 - ÉDOUARD CLAPARÈDE E HELENA ANTIPOFF}

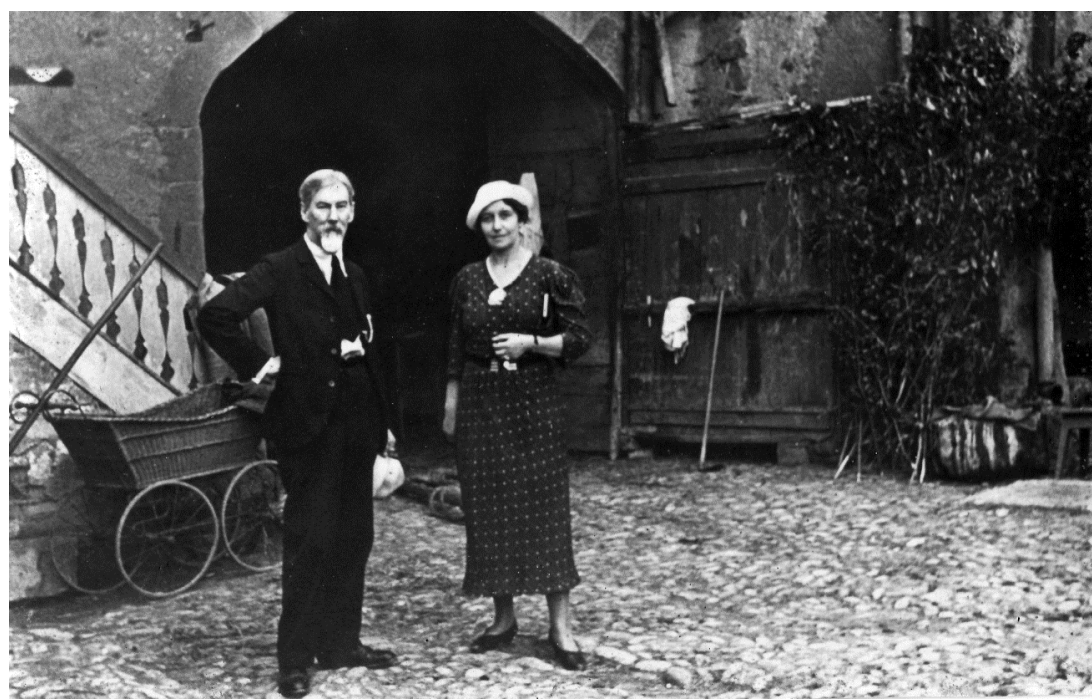

Édouard Claparède e Helena Antipoff, Belo Horizonte, Minas Gerais, Brasil, 1930.

FONTE: acervo do Centro de Documentação e Pesquisa Helena Antipoff, Belo Horizonte, MG, Brasil.

Em 1929, aceitou o convite do governo de Minas Gerais, Brasil, para atuar como professora visitante na recém-criada Escola de Aperfeiçoamento de Professores de Belo Horizonte, instituição destinada a formar educadores de elite, familiarizados com as tendências da Escola Nova preconizadas por educadores progressistas na Europa e na América do Norte, e a liderar a reforma do ensino local. Nessa Escola atuou como professora de psicologia experimental e educacional, e dirigiu o Laboratório de Psicologia recém-instalado. Por intermédio dessas atividades, colaborou na reforma do sistema educacional mineiro, em razão do incentivo à modernização de métodos e processos educativos, da coleta de dados sobre características psicológicas e psicossociais das crianças mineiras e da transmissão desses conhecimentos aos educadores, visando aumentar a eficiência de suas práticas (CAMPOS, 2010; LOURENÇO, 2001).

O contrato entre Antipoff e o governo mineiro foi sucessivamente renovado, ao longo dos anos de 1930, e nessa época Antipoff liderou a criação da Sociedade Pestalozzi de Minas Gerais e posteriormente da Sociedade Pestalozzi 
do Brasil, no Rio de Janeiro, instituições pioneiras na educação de indivíduos considerados "excepcionais", isto é, pessoas com desenvolvimento físico, psicológico ou psicossocial marcadamente diferente do desenvolvimento considerado normal em seu grupo de idade ou em seu contexto sociocultural. As Sociedades Pestalozzi e posteriormente as APAEs (Associações de Pais e Amigos dos Excepcionais), também criadas com base nesses trabalhos com excepcionais, se disseminaram pelo Brasil (DRUMOND, 2012).

Em 1940, Antipoff fundou a cadeira de Psicologia Educacional na então Universidade de Minas Gerais (atual Universidade Federal de Minas Gerais), onde contribuiu para a formação de educadores com forte formação na pesquisa em psicologia, e participou ativamente do movimento pela regulamentação da profissão de psicólogo no Brasil, ocorrida em 1962. Durante sua permanência no Brasil, Antipoff manteve contato com educadores, cientistas e intelectuais que exerceram liderança importante no movimento escolanovista e no desenvolvimento das ciências da educação na Europa.

Sabemos que as ciências da educação começaram a se estruturar como áreas de conhecimento por meio do estabelecimento dos modernos sistemas de ensino de massa em diversos países ocidentais no final do século 19, como consequência da organização dos estados-nação e das democracias modernas. A demanda social por acesso aos benefícios da educação por parte das camadas populares, associada à necessidade percebida pelas elites políticas e econômicas de fornecer padrões culturais comuns à população favoreceram a generalização da escolarização, com a oferta de instrução gratuita, pública e obrigatória. Tratava-se de permitir que todos os estudantes, quaisquer que fossem suas origens sociais, tivessem acesso à escola. Assim, na virada do século 19, milhares de estabelecimentos escolares foram construídos por toda parte, seja nas grandes ou nas pequenas cidades, especialmente nos países democráticos ocidentais. Essa escolarização em massa provocou a necessidade de expansão das instituições de formação de professores. Além disso, a gestão da heterogeneidade dos grandes grupos de crianças e jovens que chegavam às escolas tornou-se um problema para os educadores, que passaram a criticar o ensino enciclopédico e escolástico herdado de períodos anteriores, a então chamada "escola tradicional". Foi nesse contexto que nasceu o movimento chamado de "Educação Nova"4, inspirado

$4 \mathrm{O}$ movimento de educadores, intelectuais e gestores educacionais conhecido no Brasil como movimento da Escola Nova foi denominado "Éducation Nouvelle", na Europa francófona, "New Education", nos países anglo-saxões, "Movimento Progressivista", na América do Norte, Reformpädagogic, nos países germânicos, "Nueva Educación”, nos países de língua espanhola na Europa e América Latina. O movimento se iniciou de maneira espontânea em diversos países, a partir da criação de escolas experimentais com novos procedimentos pedagógicos por educadores que defendiam a renovação educacional e a construção das ciências da educação, e progressivamente 
nas propostas de pedagogos libertários dos séculos 18 e 19 - Jean-Jacques Rousseau, Johannes Heinrich Pestalozzi, Friedrich Fröbel, entre outros - e que alcançou grande influência e extensão no plano internacional a partir dos principais centros de formação em ciências da educação então estabelecidos na região central da Europa e na América. Alguns dos principais líderes desse movimento na comunidade francófona foram Alfred Binet e Édouard Claparède, que propunham um novo olhar sobre a criança e sua educação, a partir de um ponto de vista funcional. A ideia era basear a educação nas leis do desenvolvimento da criança, cuja imagem passa a ser associada ao progresso, à renovação, à esperança. (HAENGELLI-JENNI, prelo).

Édouard Claparède, mentor intelectual de Helena Antipoff desde sua formação como educadora entre 1912 e 1914, foi um líder influente do movimento da Educação Nova a partir da fundação do Instituto Rousseau, em Genebra, considerada a primeira instituição de formação em ciências da educação a ser estabelecida na Europa (HOFFSTETTER, 2010). Para o educador genebrino, a educação de sua época reclamava o desenvolvimento das ciências da educação, como forma de profissionalizar os educadores e promover a pesquisa sobre os processos de desenvolvimento infantil. Essa perspectiva inspirou a obra de Jean Piaget (1896-1980), considerada uma das mais significativas e abrangentes sobre a psicologia do desenvolvimento da cognição humana a partir da perspectiva interacionista e construtivista, com grande impacto sobre educadores e psicólogos no mundo inteiro.

Antipoff interagiu com Claparède e Piaget no Instituto Rousseau entre os anos de 1926 e 1929, e esteve em contato com ambos ao longo da vida. A correspondência que manteve com Claparède entre 1916 e 1940 foi recentemente organizada e publicada por Martine Ruchat, professora da Universidade de Genebra, e testemunha o intenso debate intelectual que ocupou os dois missivistas, evidenciando como sua interação contribuiu para orientar os trabalhos de Antipoff na Europa e no Brasil, e também para provocar a reflexão do autor genebrino sobre questões de aplicação de conceitos e teorias da psicologia em diferentes contextos culturais (RUCHAT, 2010).

se tornou internacional. O pedagogo suíço Adolphe Ferrière (1879-1960) fundou em Genebra, em 1899, o Bureau International des Écoles Nouvelles, visando reunir informações sobre o movimento. Após o fim da $1^{\mathrm{a}}$. Guerra Mundial, em 1921, foi criada a Liga Internacional da Educação Nova (LIEN) em congresso de educadores realizado em Calais, na França e aprovado o programa dos trinta pontos que definiam o que era considerado uma "escola nova" (HAENGELLI-JENNI; HOFFSTETTER, 2011). 


\section{Trabalhos de pesquisa no acervo Antipoff - traçando uma perspectiva internacional na história das ciências da educação no Brasil}

Nossos primeiros trabalhos realizados no acervo Antipoff nos revelaram a riqueza da experiência científica que continham esses documentos. Em dissertação de Mestrado, defendida em 1980 no Programa de Pós-Graduação em Educação da UFMG, eu comentava sobre a competência de Helena Antipoff ao analisar resultados de pesquisa, enfatizando as questões socioculturais que os dados revelavam (CAMPOS, 1980).

Na tese de Doutorado, defendida na Universidade de Stanford em 1989, procurei mostrar como a experiência antipoffiana se diferenciava de outras formas de abordagem das questões suscitadas pela aplicação da psicologia à educação no Brasil. Na tese, a ideia foi mostrar a tensão entre os dois projetos de escolarização que perpassavam a expansão da escola pública no Brasil: o modelo seletivo e o modelo democrático. Entre as interpretações oferecidas pela ciência psicológica para as altas taxas de fracasso escolar então observadas nas escolas públicas brasileiras entre os anos de 1930 e 1960, a explicação inatista apoiava o modelo seletivo, pois que considerava as habilidades intelectuais como dons naturais, geneticamente determinados, não modificáveis pela educação. Já as explicações ambientalistas e construtivistas, como a explicação oferecida por Helena Antipoff, consideravam que o desenvolvimento das habilidades intelectuais pela educação era não só possível como desejável, sendo uma das principais funções da escolarização. Estudei então a aplicação dessa concepção democrática da educação na experiência do Complexo Educacional da Fazenda do Rosário, dirigida e orientada por Antipoff entre os anos de 1940 e 1970, com forte influência da perspectiva defendida pela Liga Internacional da Educação Nova (os 30 pontos que definiam as escolas novas aprovados em Calais em 1921) e de Jean Piaget à frente do Bureau Internacional de Educação, em Genebra. Essa instituição intergovernamental dirigida pelo eminente psicólogo entre 1927 e 1967 tinha por finalidade precisamente contribuir na mobilização de educadores visando à construção de redes e o compartilhamento de experiências e competências que favorecessem a qualidade da educação para todos, e a paz por meio da educação (CAMPOS, 1989).

A partir desses primeiros trabalhos, participei, junto com a equipe do CDPHA então sob a coordenação do psicólogo Daniel Antipoff, do trabalho de inventário, catalogação, conservação e disponibilização do acervo para a pesquisa histórica. Durante os anos de 1990, contamos com a ajuda imprescindível da Escola de Ciência da Informação, do Centro de Conservação de Bens 
Culturais Móveis da Universidade Federal de Minas Gerais (CECOR-UFMG), e da equipe de conservação de documentos do Arquivo Público Mineiro. A documentação de que dispõe o CDPHA foi tratada com modernas técnicas de conservação pela equipe da Professora Bethânia Reis Veloso, do Laboratório de Papéis do CECOR-UFMG, como mostra a Figura 2. Com a melhor organização do acervo, foi possível realizar pesquisas mais avançadas e aprofundadas sobre diferentes aspectos da obra de Helena Antipoff e de seus colaboradores, e sobre a circulação do movimento da Escola Nova e das ciências da educação no Brasil e América Latina.

\section{FIGURA 2 - CAIXAS COM DOCUMENTOS INÉDITOS DO ACERVO DO CDPHA}

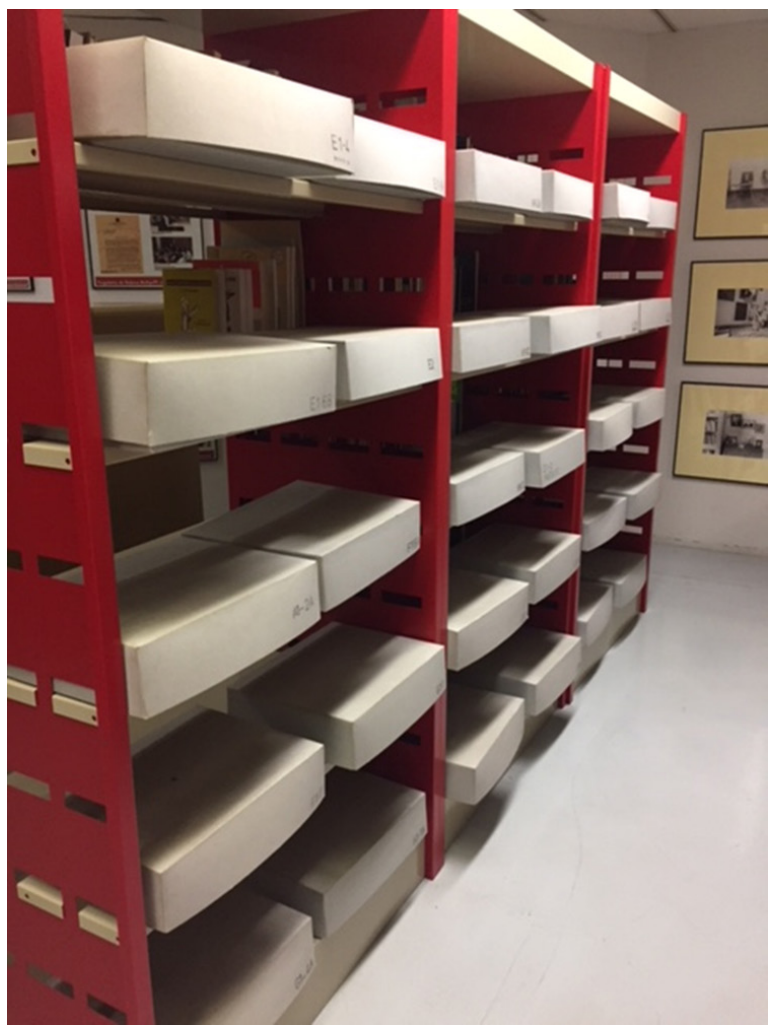

Caixas com documentos inéditos do acervo do CDPHA, confeccionadas por equipe do CECORUFMG, Sala Helena Antipoff, Biblioteca Central, Universidade Federal de Minas Gerais, Belo Horizonte, Brasil, 2018.

FONTE: acervo do Centro de Documentação e Pesquisa Helena Antipoff, Belo Horizonte, MG, Brasil. 
Nossas pesquisas se voltaram então para estudos históricos e conceituais sobre a construção, pesquisa e ensino das ciências da educação na experiência antipoffiana, e também para estudos comparados sobre alguns aspectos da psicologia infantil. Esses estudos vêm sendo feitos no quadro dos programas de pós-graduação em Educação e em Psicologia da Universidade Federal de Minas Gerais.

$\mathrm{O}$ acervo Antipoff tem nos fornecido pistas importantes para esses trabalhos historiográficos. Nas disciplinas de pós-graduação, temos trabalhado com documentos inéditos do acervo, visando iniciar os estudantes na pesquisa historiográfica ou aperfeiçoar suas habilidades na pesquisa documental.

\section{Estudos conceituais}

A observação da forte interação de Helena Antipoff com Claparède nos levou ao estudo da obra desse autor, buscando verificar quais seriam as bases de sua obra que teriam sido mais relevantes para a elaboração dos pontos de vista em psicologia e educação defendidos por Antipoff. Nassif (2008), por exemplo, estudou, de um ponto de vista internalista ${ }^{5}$, o conceito de interesse na psicologia funcional de Édouard Claparède, considerando-o o um conceito-chave para se compreender as relações entre emoção, afetividade e cognição na sua obra. A genealogia do conceito foi reconstruída, partindo das referências teóricas que o próprio autor utilizou, o que possibilitou a identificação de outros teóricos que também se preocuparam com o tema ao longo da história da psicologia e da história da psicologia da educação. $\mathrm{O}$ estudo mostra que, para Claparède, $o$ interesse aparece como a manifestação mais relevante da relação entre afetividade e cognição, constituindo uma ponte dinâmica entre o sujeito e o ambiente, ativada pela necessidade de adaptação do organismo. Essa adaptação, contudo, não é passiva, mas constitui-se em uma busca ativa de soluções para os problemas colocados pela vida em um ambiente em transformação. O estudo aponta ainda para a influência que esse ponto de vista de Claparède teria sido posteriormente retomado e aprofundado na obra de Jean Piaget (1896-1980).

5 Lakatos (1989) introduziu, nos estudos de história da ciência, a distinção entre os estudos internalistas, que se debruçam sobre a lógica de elaboração e desenvolvimento dos conceitos científicos, e os estudos externalistas, que focalizam prioritariamente as relações entre a elaboração e conceitos científicos e o contexto sociocultural em que são elaborados. 
FIGURA 3 - HELENA ANTIPOFF EM AULA

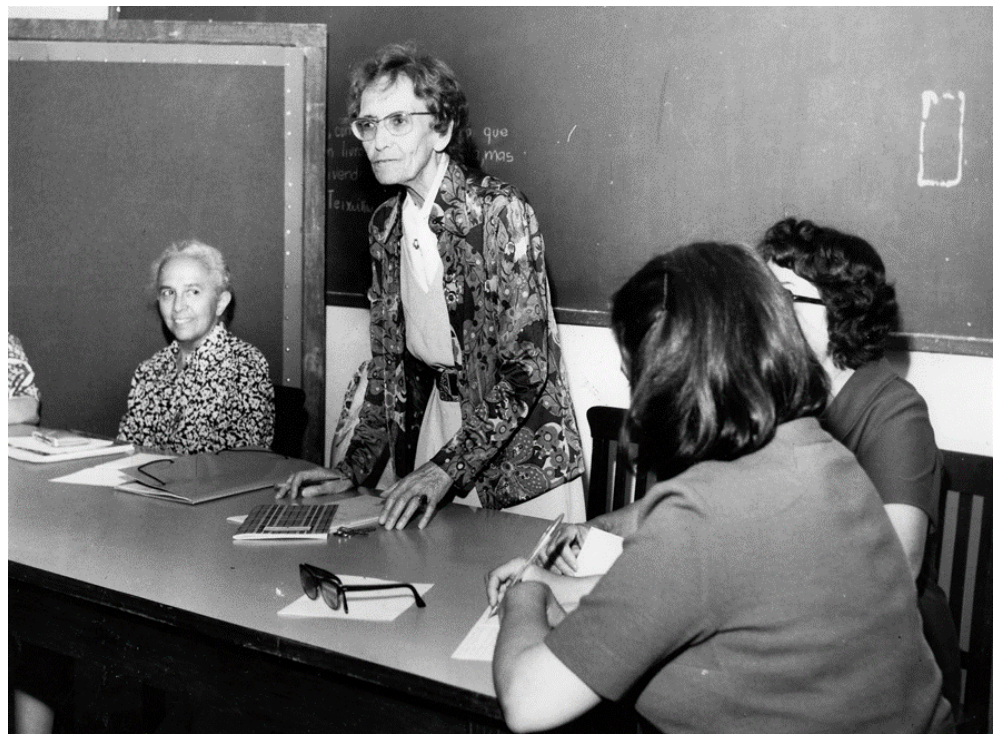

Helena Antipoff em aula, provavelmente na Sociedade Pestalozzi, Fazenda do Rosário, Ibirité, Minas Gerais, c. 1970.

FONTE: acervo do Centro de Documentação e Pesquisa Helena Antipoff, Belo Horizonte, MG, Brasil.

Outros estudos sobre o conceito de interesse foram feitos por nossa equipe, em réplicas da primeira pesquisa realizada por Antipoff em Belo Horizonte (ANTIPOFF, 2002). Nessa pesquisa, Antipoff buscou conhecer quais seriam os ideais e interesses das crianças mineiras, para melhor orientar sua educação. De seu ponto de vista, e seguindo a recomendação de Claparède, a educação escolar, para ser mais eficiente e atraente para os educandos, deveria ser guiada por seus interesses espontâneos. Associando essa perspectiva com a abordagem sociocultural da psicologia soviética, Antipoff considerava também que os interesses infantis se modificavam acompanhando as transformações sociais e culturais. Assim, a pesquisa sobre ideais e interesses deveria ser replicada a cada cinco anos para acompanhar essas modificações. A pesquisa foi realizada pela primeira vez em 1929 com uma amostra de estudantes da quarta série primária das escolas públicas de Belo Horizonte, e repetida a intervalos de cinco anos até 1944, quando o contrato de Antipoff com a Escola de Aperfeiçoamento foi encerrado. Tratava-se de um pequeno questionário de perguntas abertas, no qual as crianças deveriam relatar seus trabalhos preferidos na escola e em casa, livros 
e brinquedos prediletos, principais personagens com quem se identificavam, profissão a ser escolhida no futuro, o que fariam se tivessem muito dinheiro. As respostas eram submetidas à análise de conteúdo por categorias temáticas, tabuladas e quantificadas. Comparavam-se as respostas de meninos e meninas. $\mathrm{Na}$ interpretação dos resultados, as características do meio sociocultural em que viviam as crianças eram levadas em consideração. Realizamos várias réplicas desses estudos, a partir de 1996, introduzindo comparações também por nível socioeconômico das escolas, e comparando os resultados atuais com aqueles obtidos no passado, buscando documentar e compreender as mudanças que ocorreram na psiquê infantil no período entre 1929 e a atualidade (CAMPOS, 1996; SOUSA, 2002; GUIMARÃES, 1996; LOURENÇO; JINZENJI, 2000).

\section{Estudos sobre instituições}

O estudo das instituições onde Helena Antipoff trabalhou no Brasil e em outros países tem contribuído para a compreensão dos processos de circulação e apropriação das ciências da educação no Brasil, em especial a psicologia da educação. Essa forma de abordar a produção científica e prática dessas instituições revela muitas vezes as transformações operadas em conceitos e científicos e tecnologias quando de sua circulação em ambientes diversos. A história da ciência contemporânea observa com atenção esses processos de invenção, circulação e apropriação dos conceitos e tecnologias em diferentes contextos, sujeitos a interferências sócio-políticas, econômicas e culturais. Considera-se que o conhecimento, para ser utilizado, deve se tornar local, ou, seja, passar por transformações que o tornam suscetível de ser compreendido naquele contexto específico. Assim, na maioria das vezes, conceitos e procedimentos inventados em um contexto sofrem transformações ao serem apropriados em outro contexto, e são exatamente essas transformações que interessam ao trabalho historiográfico. São elas que permitem compreender a complexidade da interação da ciência com o contexto em que opera, e a própria lógica das decisões tomadas pelos cientistas, com grandes consequências para a chamada aplicação do conhecimento em diferentes comunidades e países. Além disso, e isso é válido especialmente no caso das ciências humanas e sociais, o processo de construção de teorias e conceitos científicos pode envolver mudanças na forma como os seres humanos pensam sobre si mesmos, sendo, portanto, um processo reflexivo, cujo impacto na interpretação do mundo operada pelos sujeitos envolvidos pode ter consequências não esperadas. Por esses motivos, o estudo da apropriação de conceitos 
e teorias em contextos diferentes de seu contexto de origem é particularmente interessante. Nesse sentido, o acervo Antipoff é especialmente relevante, por permitir acompanhar esses processos de apropriação a partir de demandas locais e das respostas elaboradas para atender essas demandas, produzindo novos conhecimentos e, eventualmente, novas interpretações da realidade. Pickren e Rutherford (2010) utilizam o conceito de indigenização para se referir a essas formas autóctones que assume o conhecimento quando apropriado em diferentes ambientes, e considera que essas formas devem legitimamente ser consideradas como parte da história da ciência, no caso da psicologia científica.

O estudo do Laboratório de Psicologia da Escola de Aperfeiçoamento de Professores de Belo Horizonte é um caso particularmente interessante para se compreender esses processos de circulação e apropriação, levando à produção de novos conhecimentos. Esse Laboratório operou como unidade de ensino e pesquisa em psicologia experimental e educacional no período entre 1929 e 1946. Nele, o ensino de psicologia era realizado com base na própria prática da pesquisa. Os levantamentos de dados realizados pelas alunas nas escolas primárias de Belo Horizonte eram ao mesmo tempo uma forma de ensino de conceitos e teorias em psicologia e também de busca de conhecimento sobre aspectos do desenvolvimento cognitivo e socioafetivo dos escolares, visando promover formas renovadas e mais eficientes de educação. Antipoff estava consciente dessa dupla função dos exercícios de laboratório, como procedimento pedagógico de ensino de psicologia e como instrumentos de conhecimento acerca da realidade local. Assim, na introdução à pesquisa sobre ideais e interesses das crianças de Belo Horizonte, Antipoff cita os motivos para realizá-la: a necessidade de conhecer melhor as crianças locais e de formar as alunas nos métodos da psicologia experimental:

Convidada pelo Governo de Minas Gerais para organizar um laboratório de psicologia pedagógica, na Escola de Aperfeiçoamento, para as professoras do Estado, e de promover investigações entre os alunos, a fim de estabelecer as normas de desenvolvimento físico e mental, procurei, logo depois de chegada a um país totalmente desconhecido para mim, encontrar uma sonda que me permitisse orientar-me, o mais depressa possível, quanto à psicologia dos pequenos brasileiros, e apanhar a sua fisionomia psíquica geral. [...] Tendo como colaboradoras alunas da Escola de Aperfeiçoamento, eu era obrigada não somente a ter em vista o fim científico [...] como também o fim prático de iniciar e preparar minhas alunas nos métodos da psicologia experimental. (ANTIPOFF, 2002, p. 133). 
Estudos já feitos sobre esse laboratório mostram que ele efetivamente funcionou como unidade de ensino e pesquisa em psicologia e educação. Miranda (2014) afirma que o laboratório foi ferramenta importante no ensino de psicologia, porque permitiu a realização de estudos psicológicos que contribuíram para a formação prática das estudantes-professoras, proporcionando a construção de conhecimentos sobre as escolas locais, a infância brasileira e as professoras da época. Também Miranda e Cirino (2015) afirmaram que o laboratório desempenhou papel importante na produção de conhecimentos psicológicos sobre a educação elementar no Brasil da época, fornecendo um modelo para a organização da pesquisa em psicologia no país a partir de seu ensino.

\section{FIGURA 4 - ORIENTAÇÃO DE ALUNAS POR HELENA ANTIPOFF}

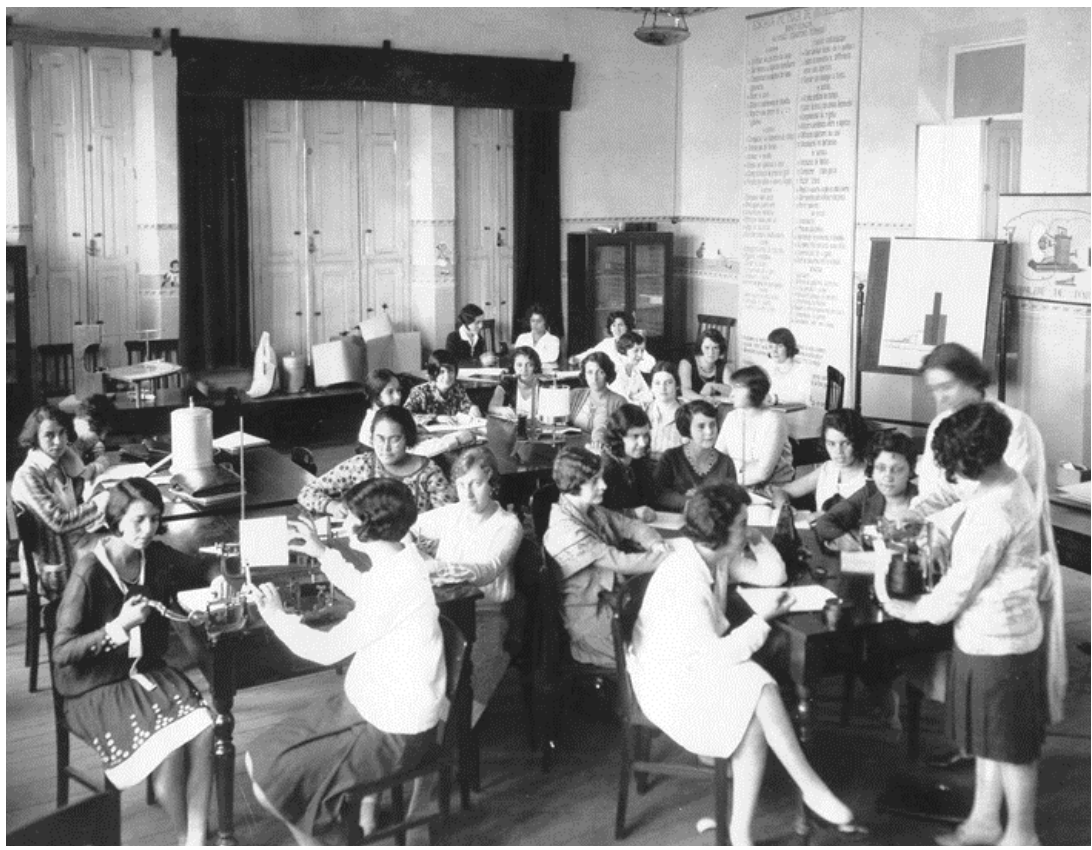

Helena Antipoff (de pé, de avental branco, à direita) orienta alunas em pesquisas no Laboratório de Psicologia da Escola de Aperfeiçoamento de Professores de Belo Horizonte, Belo Horizonte, Brasil, c. 1930.

FONTE: acervo do Centro de Documentação e Pesquisa Helena Antipoff, Belo Horizonte, MG, Brasil. 
Uma outra instituição fundada sob a liderança de Helena Antipoff foi a Sociedade Pestalozzi de Minas Gerais, criada em 1932 por um grupo de intelectuais, professores e religiosos com o objetivo de cuidar da educação e assistência a crianças e adolescentes excepcionais e em situação de risco social. Antipoff, como professora na Escola de Aperfeiçoamento de Belo Horizonte, vinha observando a quase absoluta ausência, na cidade, de equipamentos educacionais e sociais direcionados a esse grupo. Inspirada na Declaração Internacional dos Direitos da Criança aprovada pela Liga das Nações em 1924, chamou a atenção das elites de Belo Horizonte para a necessidade de providenciar instituições para o diagnóstico, tratamento e educação dos excepcionais, tanto os portadores de distúrbios orgânicos quanto dos "excepcionais sociais", oriundos de famílias de baixa renda. A Sociedade Pestalozzi deu origem a diversas instituições, entre as quais se destacam o Instituto Pestalozzi, destinado ao cuidado médico, psicológico e pedagógico dos excepcionais, e a Fazenda do Rosário, fundada em 1940, complexo educacional para a educação e profissionalização dos excepcionais, e para a formação e especialização de educadores.

Estudos têm sido feitos sobre diversos aspectos da atuação das instituições ligadas à Pestalozzi. Vieira (2008) examinou a trajetória da Clínica de Psicologia Édouard Claparède, localizada na Fazenda do Rosário, entre 1955 e a atualidade. Estudando o funcionamento da Clínica com base em uma amostra de laudos psicológicos, Vieira mostra as características inovadoras e atuais do trabalho ali desenvolvido por um grupo de profissionais conectado com as questões socioculturais que atravessam os diagnósticos psicológicos. Também Borges (2014) realizou pesquisa sobre os laudos psicológicos produzidos no Instituto Pestalozzi, em Belo Horizonte, a partir dos anos de 1930, evidenciando que a prática da psicologia no Instituto, sob a orientação de Helena Antipoff, já anunciava procedimentos que hoje denominamos inclusivos. No Pestalozzi, a avaliação das características singulares de cada sujeito no processo de aprendizagem levava a uma compreensão mais aprofundada de suas dificuldades e a alternativas educacionais inovadoras e favoráveis ao desenvolvimento das potencialidades do educando. Experiências atuais derivadas da tradição antipoffiana também têm sido estudadas, como a Escola EDUC - Centro de Educação Criadora, fundada por Daniel e Ottília Antipoff nos anos de 1970, com proposta pedagógica humanista e libertária voltada para a educação de bem-dotados (ANTIPOFF, 2017).

A partir da constatação do valor das experiências de aplicação da psicologia a questões educacionais orientadas por Helena Antipoff, pautadas na busca genuína de melhores condições para o tratamento dos problemas de aprendizagem no contexto escolar, passamos a estudar também o ensino de psicologia e das ciências da educação na formação de professores, sob a influência da educadora, e estes são os trabalhos que nos ocupam na atualidade. Recentemente foi defendida 
a tese de Adriana Duarte (2017) sobre o ensino de psicologia na formação de professores na experiência do Instituto Superior de Educação Rural, na Fazenda do Rosário, nos anos de 1950 e 1960. Evidenciou-se, no estudo, que o modelo experimentado no Laboratório de Psicologia da Escola de Aperfeiçoamento de Professores de Belo Horizonte nos anos de 1930 foi reproduzido e ampliado na Fazenda do Rosário, sendo o ensino de psicologia centrado na relação teoria e prática e na pesquisa. Cassemiro (2018) pesquisou sobre a formação de professores para a educação especial na experiência do Rosário, mostrando também as características humanistas dessa formação, associadas à sólida formação em teoria e pesquisa. Esses trabalhos têm sido feitos a partir de registros de práticas pedagógicas na formação de educadores encontrados em preciosa coleção de diários que fazem parte do acervo do CDPHA. Os diários relatam as atividades de estudantes e professores no Complexo Educacional da Fazenda do Rosário nas décadas de 1940 a 1970.

\section{Considerações finais}

Os trabalhos de pesquisa que temos produzido a partir do acervo documental do Centro de Documentação e Pesquisa Helena Antipoff têm contribuído, a nosso ver, para iluminar um capítulo importante da história das ciências da educação no Brasil, com dimensão internacional. O trabalho de Helena Antipoff como pesquisadora e as inovações teórico-práticas que introduziu nas áreas da psicologia e da educação têm sido reconhecidos por sua originalidade e consistência. A síntese que operou entre diversas correntes de pensamento em psicologia e educação com as quais dialogou é atualmente considerada contribuição de grande relevância para o desenvolvimento da psicologia e da educação contemporâneas (CAMPOS, 2012; CAMPOS; BORGES, 2017).

Por outro lado, as experiências educativas, inovações teórico-práticas e institucionais que estudamos têm mostrado formas alternativas de tratar questões de grande atualidade no contexto educacional, que podem inspirar os trabalhos de educadores comprometidos com o aperfeiçoamento e desenvolvimento da educação contemporânea. 


\section{REFERÊNCIAS}

ANTIPOFF, C. A. A Escola EDUC - Centro de Educação Criadora: uma proposta pedagógica humanista e ecológica no contexto das transformações da educação contemporânea. 2017. Tese (Doutorado em Educação) - Universidade Federal de Minas Gerais, Belo Horizonte, 2017.

ANTIPOFF, H. Ideais e interesses das crianças de Belo Horizonte e algumas sugestões pedagógicas. In: CAMPOS, R. H. F. (Org.) Helena Antipoff: Textos Escolhidos. São Paulo: Casa do Psicólogo; Brasília: Conselho Federal de Psicologia, 2002, p. 133-157. (Coleção Clássicos da Psicologia Brasileira).

ASSIS, R. M.; LOURENÇO, É; BORGES, A. (Org.) Cultura, direitos humanos e práticas inclusivas em psicologia e educação. Belo Horizonte: CDPHA, Editora PUC Minas, 2015. (Coleção Encontros Anuais Helena Antipoff).

BORGES, A. A. P. Entre tratar e educar os excepcionais: Helena Antipoff e a psicologia na Sociedade Pestalozzi de Minas Gerais (1932-1942). 2014. Tese (Doutorado em Educação) - Universidade Federal de Minas Gerais, Belo Horizonte, 2014.

CAMPOS, R. H. F. Psicologia e ideologia: um estudo da formação da psicologia educacional em Minas Gerais. 1980. Dissertação (Mestrado em Educação) - Universidade Federal de Minas Gerais, 1980.

CAMPOS, R. H. F. Conflicting interpretations of intelectual abilities among Brazilian psychologists and their impact on primary schooling. 1989. Tese (PhD em Educação). Stanford University, Stanford, EUA, 1989.

CAMPOS, R. H. F. Impacto de transformações socioculturais no imaginário infantil (1929-1993). Psicologia e Sociedade, v. 8, n. 2, p. 45-62, 1996.

CAMPOS, R. H. F. Helena Antipoff: uma biografia intelectual. Rio de Janeiro: Fundação Miguel de Cervantes, 2012.

CAMPOS, R. H. F.; VIEIRA, R. C. (Org.). Instituições e psicologia no Brasil. Rio de Janeiro, RJ: Nau Editora, 2007. (Coleção Encontros Anuais Helena Antipoff).

CAMPOS, R. H. F.; DUARTE, A. O. S. A.; CASSEMIRO, M. F. P. (Org.). Psicologia, educação e o debate ambiental: questões históricas e contemporâneas. Belo Horizonte: FAE/UFMG; CDPHA, 2017.

CAMPOS, R. H. F.; BORGES, A. A. P. De Genève à Belo Horizonte, une histoire croisée: circulation, réception et réinterprétation d'un modèle Européen des classes spéciales au Brésil des années 1930. In: DROUX, Joëlle; HOFFSTETTER, Rita (Eds.) Border crossing in Education: Historical perspectives on transnational connections and circulations. London, New York: Routledge, 2017, p. 193-210.

CASSEMIRO, M. F. P. Formação de professores para a educação especial: a experiência 
de Helena Antipoff e seus colaboradores na Fazenda do Rosário (1940-1971). 2018. Tese (Doutorado em Educação) - Universidade Federal de Minas Gerais, 2018.

DRUMOND, A. C. A Associação de Pais e Amigos dos Excepcionais de Barbacena e o atendimento às pessoas com Síndrome de Down (1962-1974): diálogos com pestalozzianos. 2012. Tese (Doutorado em Educação) - Universidade Federal de Minas Gerais, 2012.

DUARTE, A. O. Psicologia na formação de professores: interligação entre teoria e prática nos cursos da Fazenda do Rosário, Ibirité, Minas Gerais (1948-1974). 2017. Tese (Doutorado em Educação) - Universidade Federal de Minas Gerais, 2017.

GUIMARÃES, M. C. F. Interesses infantis por atividades na escola e em casa: uma contribuição ao trabalho de Antipoff. 1996. Dissertação (Mestrado em Psicologia) Universidade Federal de Minas Gerais, 1996.

GUTIERREZ, L. Histoire du mouvement de l'éducation nouvelle. Carrefours de l'Éducation 31, p. 5-9, Janvier-Juin 2011.

HAENGGELI-JENNI, B. Pour l'Ere Nouvelle: une revue au carrefour entre science et militance (1922-1940). (s.d.). Thèse en cours de publication; Peter Lang.

HAENGELLI-JENNI, B.; HOFFSTETTER, R. Pour l'Ere Nouvelle (1922-1940). La Science convoquée pour fonder une "internationale de l'éducation". Carrefours de l'Éducation 31, p. 105-136, Janvier-Juin 2011.

HOFFSTETTER, R. Genève: creuset des sciences de l'éducation. Genève: Droz, 2010.

LAKATOS, I. La metodología de los programas de investigación científica. Madrid: Alianza, 1989.

LOURENÇO, E.; JINZENJI, M. Y. Ideais das crianças mineiras no século XX: mudanças e continuidades. Psic.: Teor. e Pesq., v. 16, n. 1, p. 41-48, abr. 2000.

LOURENÇO, E. Contribuição à historiografia da psicologia: a psicologia da educação na obra de Helena Antipoff. 2001. Dissertação (Mestrado em Psicologia) - Universidade Federal de Minas Gerais, 2001.

LOURENÇO, E.; GUEDES, M. C.; CAMPOS, R. H. F. (Org.). Patrimônio cultural, museus, ciência e educação: diálogos. Belo Horizonte, MG: PUC Minas, 2009. (Coleção Encontros Anuais Helena Antipoff).

LOURENÇO, E.; ASSIS, R. M.; CAMPOS, R. H. F. (Org.). História da Psicologia e Contexto Sociocultural. Belo Horizonte, MG: Editora PUC Minas, 2012. (Coleção Encontros Anuais Helena Antipoff).

MIRANDA, R. O Laboratório de Psicologia da Escola de Aperfeiçoamento de Professores de Belo Horizonte: diálogos entre Psicologia e Educação (1929-1946). 2014. Tese (Doutorado em Educação) - Universidade Federal de Minas Gerais, Belo Horizonte, 2014.

MIRANDA, R. L.; CIRINO, S.; The role of a laboratory of experimental psychology in the Brazilian education renewal of the 1930s. History of Psychology, Vol 18(1), Feb 
2015, 69-77. Disponível em: <http://dx.doi-org.ez27.periodicos.capes.gov.br/10.1037/ a0038446>. Acesso em: 28 mar. 2018.

NASSIF, L. Édouard Claparède: da chave biológica à interpretação interacionista da vida mental. 2008. Tese (Doutorado em Educação) - Universidade Federal de Minas Gerais, Belo Horizonte, 2008.

NASSIF, L.; NUNES, M. T. Formação de professores: diálogos com a experiência antipoffiana. Belo Horizonte: Editora PUC Minas, 2008.

PICKREN, W.; RUTHERFORD, A. A History of Modern Psychology in Context. Hoboken, NJ, USA: John Willey \& Sons, 2010.

RUCHAT, M. Édouard Claparède - Hélène Antipoff: Correspondance (1914-1940). Firenze: Leo Olshki Editore, 2010.

SANTIAGO, A. L.; CAMPOS, R. H. F. (Org.). Educação de crianças e jovens na contemporaneidade: pesquisas sobre sintomas na escola e subjetividade. Belo Horizonte, MG: PUC Minas, 2011. (Coleção Encontros Anuais Helena Antipoff).

SOUSA, M. C. M. A contemporaneidade de Helena Antipoff na fala dos meninos e meninas do sertão. 2002. Dissertação (Mestrado em Educação), Universidade Federal de Minas Gerais, Belo Horizonte, 2002.

VIEIRA, R. C. O psicólogo e seu fazer na Educação: contando uma outra história. 2008. Tese (Doutorado em Educação) - Universidade Federal de Minas Gerais, 2008.

Texto recebido em 17 de maio de 2018 . Texto aprovado em 17 de julho de 2018. 